\title{
AVALIAÇÃO DA FORÇA MUSCULAR RESPIRATÓRIA EM INDIVÍDUOS COM SÍNDROME DE DOWN
}

\author{
Cássia Pereira da Silva \\ Graduando em Fisioterapia do UNIFOR-MG \\ Bolsista do CNPq/FAPEMIG \\ E-mail:cassiasilvaptamg@ hotmail.com \\ Ywia Danieli Valadares \\ Professora no UNIFOR-MG \\ Orientadora no Programa de Iniciação Científica do UNIFOR-MG/CNPq/FAPEMIG \\ E-mail: ywiaval@hotmail.com
}

Recebido em: 12/10/2014

Aprovado em: 22/10/2014

\section{RESUMO}

A Síndrome de Down é a forma mais frequente de deficiência mental causada por uma alteração cromossômica microscópica que acarreta maiores chances de doenças associadas, como os problemas relacionados ao sistema respiratório. Decorrente a esse fator tal estudo objetivou-se avaliar a força muscular respiratória em indivíduos com Síndrome de Down, bem como comparar os valores medidos de Pressão inspiratória máxima (Pi max.) e Pressão expiratória máxima (Pe max.) com os preditos para idade e gênero. Realizado no período de março de 2013 á fevereiro de 2014, com 19 indivíduos portadores de Síndrome de Down, com idades entre 8 e 46 anos, selecionados através de um banco de dados, disponibilizado pelas APAES dos municípios de Pimenta-MG e Formiga-MG, mediante consentimento dos pais, os voluntários foram medidos e pesados para posterior cálculo do índice de massa corporal, foram submetidos à avaliação de força muscular respiratória através do manuvacuômetro. Quando se comparou a Pi Max prevista com a PI max obtida e a Pe max prevista com a Pe max obtida observou-se diferença significativa $(\mathrm{p}=0,000)$. Nas correlações das variáveis: IMC, Idade com Pi max obtida, Pe max obtida, observou correlação apenas entre as variáveis IMC e Pe max obtida. Com os resultados aqui obtidos enfatiza-se a importância da criação de programas de fisioterapia cardiorrespiratória para essa população a fim de prevenir possíveis complicações e instalações de patologias, a fim de reabilitar a perda funcional já instalada, e assim também proporcionar uma melhor qualidade de vida para essa população.

Palavras-chave: Força muscular respiratória. Síndrome de Down.

\section{ASSESSMENT OF RESPIRATORY MUSCLE STRENGTH IN INDIVIDUALS WITH DOWN SYNDROME}




\begin{abstract}
Down syndrome is the most frequent form of mental retardation caused by a microscopic chromosomal alteration that results in higher chances of associated diseases, such as problems related to the respiratory system. Due to such factors, the study aimed to evaluate respiratory muscle strength in individuals with Down syndrome, and to compare the measured values of maximal inspiratory pressure ( $\mathrm{Pi}$ max) and maximal expiratory pressure ( $\mathrm{Pe}$ max) with predicted age and gender. Conducted from March 2013 to February 2014, with 19 individuals with Down Syndrome, aged between 8 and 46 years, selected through a database provided by the municipalities APAES of Pimenta-MG and Formiga-MG upon parental consent, volunteers were measured and weighed for subsequent calculation of body mass index, underwent assessment of respiratory muscle strength by manometer. When comparing the Pi max provided with Pi max obtained and the Pe max provided with Pe max obtained showed a significant difference $(\mathrm{p}=0.000)$. The correlations of the variables: BMI, Age with Pi max obtained, Pe max obtained only observed correlation between BMI and Pe variables max retrieved. As the results obtained here emphasize the importance of establishing cardiopulmonary physiotherapy programs for this population in order to prevent possible complications and pathology facilities in order to rehabilitate the already established functional loss, and so provide a better quality of life for this population.
\end{abstract}

Keywords: Respiratory muscle strength. Down Syndrome.

\title{
1 INTRODUÇÃO
}

Descrita por um médico britânico, a Síndrome de Down (SD) também denominada de Trissomia do Cromossomo 21, é a forma mais frequente de deficiência mental causada por uma alteração cromossômica microscópica sendo a trissomia cromossômica a alteração com maior número de ocorrência, que leva ao retardo do desenvolvimento, características físicas peculiares e uma redução intelectual, assim como a presença de cardiopatias e maior incidência de infecções respiratórias e más formações congênitas (GRANZOTTI et al., 1995; SILVA; DESSEN, 2002).

A SD tem origem durante a formação dos gametas reprodutivos ou em seguida á fecundação, onde ocorre um erro na separação dos cromossomos 21, durante a meiose ou mitose (BERTHOLD et al., 2004).

Pode ser diagnosticada por meio da realização da cariotipagem cromossômica das microvilosidades coriônicas ainda no período gestacional, no entanto ainda prevalece o diagnóstico por meio das características fenotípicas descritas durante ou imediatamente após o nascimento (SCHWARTZMAN, 2003).

Pouco se sabe em relação às causas que poderiam aumentar a incidência do aparecimento da $\mathrm{SD}$, porém vários autores citam duas classes, dentre elas: as causas endógenas e as exógenas, dentre as endógenas a mais importante é a relação da idade 
gestacional, onde nota-se uma maior prevalência de casos entre as gestantes acima de 35 anos de idade, além de mães portadoras de problemas da tireoide (GUSMÃO; TAVARES; MOREIRA, 2003; SCHWARTZMAN, 2003).

Já em relação às causas exógenas estudos afirmam existir uma diminuição dos índices da síndrome com a realização de diagnósticos pré-natais, a radiação, e o uso de pílulas anticonceptivas que aumentaria a incidência da síndrome (SCHWARTZMAN, 2003).

A SD tem aumentado na população em geral isso seria em consequência do aumento da expectativa de vida assim como do aprimoramento de técnicas de tratamento, em especial a estimulação precoce com fisioterapia e outras modalidades multidisciplinares, que irão proporcionar um melhor desenvolvimento e desempenho social de indivíduos portadores da SD (MOREIRA; EL-HANI; GUSMÃO, 2000).

Os indivíduos portadores da SD possuem diversas alterações das características fenotípicas e funcionais, assim como dos níveis variáveis de deficiência mental (BERTHOLD, 2004).

Dentre as alterações fenotípicas citam-se as oculares apresentando presença da fissura palpebral oblíqua, um alargamento da prega epicântrica, patologias visuais como o estrabismo e catarata congênita (BERTHOLD, 2004).

Ocorrem também alterações no pescoço, que geralmente é mais largo, curto e apresenta uma prega cutânea na região da nuca. As mãos possuem o diâmetro látero-lateral maior que o crânio-caudal, os dedos são curtos e grossos, o quinto dedo pode apresentar a clinodactilia e presença de prega palmar única (BERTHOLD, 2004).

As orelhas apresentam má formação, com lóbulos pequenos ou ausentes, e implantação mais baixa que a normalidade. Sua audição tem maiores chances de comprometimento, pois seu conduto interno é mais estreito, podendo ocorrer até sua perda. Assim como alterações gastrointestinais e da cavidade oral (BERTHOLD, 2004).

Além das características peculiares presentes no sistema cardiovascular, como a permanência do canal atroventricular, que ocorre em $60 \%$ dos casos, defeito isolado do septo ventricular, e/ou septo atrial, ductus arteriosus patente (30\%), tetralogia de Fallot (7\%), defeitos isolados das válvulas e coartação da aorta (SCHWARTZMAN, 2003).

$\mathrm{E}$ as alterações respiratórias e pulmonares, ocorrendo geralmente a obstrução das vias aéreas, devido algum tipo de corpo obstrutivo ou hipotonia da musculatura da faringe, devido dimensões reduzidas da traqueia, ou o aumento de secreções, que irão facilitar a apnéia do sono, a hipoxemia e a hipertensão pulmonar geralmente associadas a SD. Ambos estes fatores complicantes são predisponentes para a maior mortalidade nesse grupo populacional, 
principalmente em crianças antes de um ano de idade, que irão comprometer a biomecânica respiratória e consequentemente as trocas gasosas e o aporte de oxigênio disponibilizado para todos os órgãos e tecidos (SCHWARTZMAN, 2003).

Por se tratar de uma doença com possíveis disfunções associadas ao sistema cardiorrespiratório e, consequentemente, prejudicando a qualidade de vida desses indivíduos, a avaliação das forças respiratórias se torna necessária para que seja possível quantificar as consequências das principais alterações decorrente da Síndrome para o paciente e preparar estratégias preventivas. Sendo assim, este estudo objetivou avaliar a força muscular respiratória (Pi max e Pe max) por meio do manuvacuômetro. Assim como comparar os valores medidos de Pi max e Pe max e IMC com os preditos para sua idade e gênero.

\section{METODOLOGIA}

Inicialmente foi realizado contato com a secretária de saúde da cidade de Formiga-MG e Pimenta-MG, para apresentação do projeto e autorização para execução do mesmo. Após a aprovação do comitê de ética em pesquisa em seres humanos do Centro Universitário de Formiga (UNIFOR-MG) foi iniciado o protocolo. Primeiramente, a pesquisadora realizou visitas domiciliares a fim de se informar a respeito do estudo e mediante a assinatura do termo de consentimento livre esclarecido e termo de assentimento foi iniciada a coleta de dados.

Após a definição amostral, os participantes foram medidos e pesados para cálculo do IMC. Finalizando foi aplicado o teste de força muscular respiratória Pi max e Pe max. O clipe nasal foi utilizado para evitar o escape de ar pelas vias aéreas superiores e os voluntários permaneceram sentados. Previamente os voluntários foram orientados através de toques proprioceptivos no abdome, quanto ao procedimento da Pi max. Consequentemente foram realizadas três medidas de teste, com a pesquisadora realizando estímulos verbais padronizados. Após, foi iniciada a coleta de três medidas de Pi max, sendo que deveriam permanecer dentro de uma diferença de $5 \%$ ou 10 pontos entre elas. Caso houvesse variação, outras medidas seriam realizadas, sendo coletadas no máximo cinco medidas. A maior medida foi considerada, o mesmo procedimento foi realizado para a Pe max.

Os critérios de inclusão foram:

- Idade entre 8 e 59 anos;

- Diagnóstico médico da SD;

- Os participantes concordarem com o estudo e assinarem o Termo de Assentimento; 
- Os pais ou responsáveis concordarem com o estudo e assinarem o TCLE.

Os critérios de exclusão:

- Diagnóstico médico de doença cardiovascular, respiratória obstrutiva ou restritiva;

- Indivíduos tabagistas;

- Instabilidade hemodinâmica no momento da coleta (avaliada através da medida da PA);

- Não realização de alguma das avaliações do protocolo.

Foi realizada a análise descritiva dos dados, estando estes apresentados sob média e desvio padrão na forma de gráficos e tabelas.

Em seguida foram realizados os testes estatísticos, onde o Kolmogorov smirnov avaliou a normalidade dos dados, sendo estes paramétricos utilizou-se o teste t para comparar a força muscular respiratória atingida com a prevista para a idade e sexo dos voluntários.

Utilizou-se o software Mini tab e $\mathrm{p}<0,05$.

\section{RESULTADOS}

A população inicial foi composta por 22 indivíduos, com diagnóstico médico de Síndrome de Down e destes, 3 foram excluídos devido a não compreensão dos comandos verbais solicitados pela aluna para realização dos testes, totalizando uma amostra final de 19 voluntários.

Dos 19 avaliados, $14(63,63 \%)$ eram do gênero masculino e 5 (36,37\%) do feminino. A idade variou entre 8 e 46 anos com média de 23,84 \pm 9,77 anos, sendo 19 (100\%) solteiros.

O IMC variou entre 15,93 e $40\left(\mathrm{~kg} / \mathrm{m}^{2}\right)$, média de $25,42 \pm 6,48\left(\mathrm{Kg} / \mathrm{m}^{2}\right)$.

As médias e desvio padrão da Pi max prevista e obtida, Pe max prevista e obtida, estão demonstradas na TAB. 1. 
Tabela 1 - Pi max e Pe max prevista e obtida

\begin{tabular}{|c|c|c|c|c|}
\hline & $\begin{array}{l}\text { Pi max } \\
\text { prevista } \\
(\mathrm{cmH2O})\end{array}$ & $\begin{array}{l}\text { Pi max } \\
\text { obtida } \\
\text { (cmH2O) }\end{array}$ & $\begin{array}{l}\text { Pe max } \\
\text { prevista } \\
(\mathrm{cmH2O})\end{array}$ & $\begin{array}{l}\text { Pe max } \\
\text { obtida } \\
\text { (cmH2O) }\end{array}$ \\
\hline \multirow[t]{2}{*}{ Variação } & $-88,7$ e - & $-11 \mathrm{a}$ & 88,19 & 25 e 80 \\
\hline & 153,57 & 114 & 259,76 & \\
\hline Média \pm & $123,24 \mathrm{e}$ & $-41,42 \pm-$ & $217,11 \pm$ & 49,94 \\
\hline DV & $\pm-17,03$ & 28,52 . & 47,20 & 17,94 . \\
\hline
\end{tabular}

Fonte: Dados da pesquisa.

Quando se comparou a Pi max prevista com a Pi max obtida observou-se diferença significativa $(\mathrm{p}=0,000)$ entre as variáveis, conforme visto no GRAF. 1. Assim como também foi observada diferença significativa nas comparações da Pe max prevista com os valores da Pe max obtida conforme observado no GRAF. 2.

Gráfico 1 - Comparação dos valores previstos e valores obtidos na avaliação da Pi max: Pressão inspiratória máxima

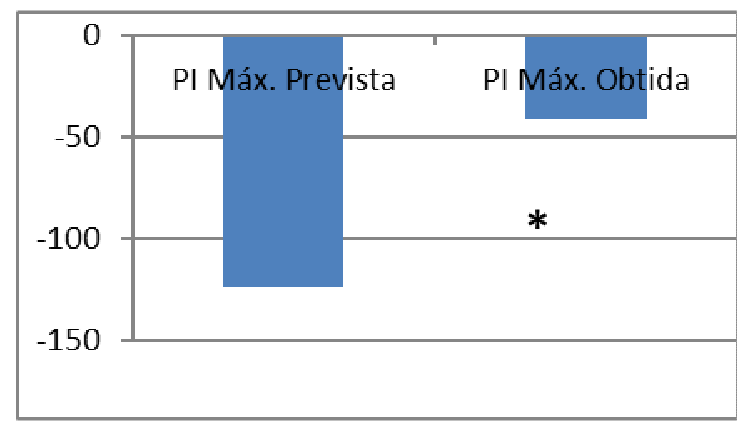

Fonte: Dados da pesquisa. 
Gráfico 2 - Comparação dos valores previstos e valores obtidos na avaliação da Pe max: Pressão expiratória máxima

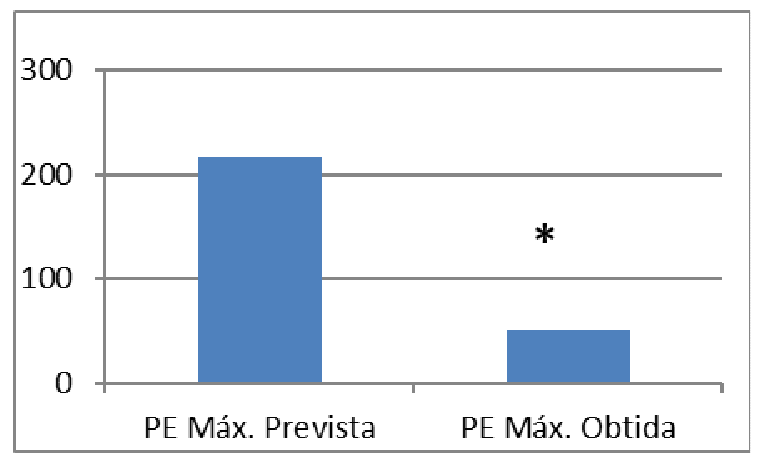

Fonte: Dados da pesquisa.

Quando se correlacionou os dados: IMC, IDADE com Pi max obtida, Pe max obtida, observou-se correlação apenas entre as variáveis IMC e Pe max obtida, como demonstra a TAB. 2.

Tabela 2 - Coeficientes de correlação (R) das variáveis: Pi max obtida, Pe max obtida, IMC, IDADE

\begin{tabular}{lll}
\hline & Pi max & Pe max \\
& Obtida & Obtida \\
& & \\
\hline IMC & 0,33 & $0,47^{*}$ \\
\hline IDADE & 0,27 & \\
& \\
\hline \\
Fonte: Dados da pesquisa. \\
Nota: $*$ p $<0,05$
\end{tabular}

\section{DISCUSSÃO}

No presente estudo foram excluídos indivíduos com diagnóstico médico de doença cardiovascular, respiratória obstrutiva ou restritiva, pois segundo pesquisa realizada as patologias obstrutivas do aparelho respiratório acarretam várias consequências para o organismo que consequentemente irá prejudicar a funcionalidade da musculatura respiratória, diminuindo assim sua força (AQUINO et al., 2010). 
Já o parâmetro de exclusão que englobou as doenças cardiovasculares partiu da análise do ponto em que tais doenças levam a uma deficiência do bombeamento de sangue pelo coração o que consequentemente comprometeria a capacidade pulmonar, principalmente a desenvoltura biomecânica da musculatura respiratória (JUNIOR et al., 2007).

Também foram excluídos do presente estudo indivíduos tabagistas, pois as substâncias presentes na composição do cigarro principalmente a nicotina e o monóxido de carbono geram alterações morfológicas em praticamente todos os tecidos corporais, comprometendo principalmente e mais intensamente os tecidos do sistema cardiorrespiratório. O primeiro a sofrer com as ações lesivas do tabaco é o epitélio do trato respiratório alterando sua função e constituição, a inalação da fumaça do cigarro também está relacionada com alterações na produção de muco, além de prejudicar o transporte mucociliar, aumentando o risco do desenvolvimento de doenças respiratórias (TAMASHIRO et al., 2009).

Também foram excluídos indivíduos que apresentaram instabilidade hemodinâmica no momento da coleta, analisada através da aferição da pressão arterial no ato da coleta dos dados, pois as alterações cardiovasculares estão intimamente relacionadas com a funcionalidade e capacidade hemodinâmica do transporte do oxigênio, comprometendo as pressões pulmonares podendo acrescentar vieses a pesquisa e diminuindo sua veracidade (TAMASHIRO et al., 2009).

No estudo foi pré-determinada a faixa etária para coleta de dados de 8 a 59 anos, foi utilizada a idade limítrofe de 8 anos, com a finalidade de realizar uma maior abrangência dos indivíduos que estão na fase escolar, assim como pelo fato de vários estudos relatados sugerirem que $88 \%$ das crianças portadoras de Síndrome de Down possivelmente serão hospitalizadas em algum momento antes dos 16 anos, devido alguma complicação respiratória, havendo assim a necessidade da análise das condições da força muscular respiratória desta faixa etária (TAMASHIRO et al., 2009).

Também foi determinada tal faixa decorrente da idade de maturação do sistema respiratório onde o desenvolvimento de tal sistema está dividido em cinco fases sendo estas: embrionária, pseudoglandular, canalicular, sacular e alveolar, onde o desenvolvimento continua até a primeira infância que compreende ás idades de 6 a 7 anos. No outro extremo concordou-se em utilizar 59 anos de idade, pois com o avançar da idade alterações características do envelhecimento poderiam acrescentar vieses à pesquisa, sendo que a faixa etária que compreende acima dos 60 anos de idade é caracterizada por uma progressiva diminuição da capacidade cardiorrespiratória, mobilidade, força e resistência muscular, que 
estão relacionadas com uma alteração denominada sarcopenia que compreende a diminuição da força e volume muscular (RUIVO; VIANA; MARTINS; BAETA, 2009).

A variação da idade encontrada no presente estudo está de acordo com achados na literatura, onde a taxa de maior sobrevida de portadores de SD está até os 30 anos de idade sendo esta taxa de 79\% (SCHWARTZMAN, 2003).

A predominância do gênero masculino sobre o feminino observada no estudo pode ser justificada pela maior proporcionalidade de tal gênero sobre o feminino na lista prévia, disponibilizada pela APAE do município de Formiga, Minas Gerais.

Em concordância com estes, outros estudos também observaram uma maior predominância do gênero masculino, sendo que esta ocorre com uma maior proporcionalidade em portadores de SD do que a observada na população em geral, onde notasse os valores de 1,27: 1 (SCHWARTZMAN, 2003).

Ao se analisar o IMC dos indivíduos obteve-se uma variação entre 15,93 e 40 (kg/m²), média de 25,42 $\pm 6,48\left(\mathrm{Kg} / \mathrm{m}^{2}\right)$, partindo-se dos valores medianos tais indivíduos estão classificados em estado de sobrepeso, o que concorda com estudos, onde relatam uma maior prevalência de deposição de tecido adiposo em indivíduos com Síndrome de Down (SILVA et al, 2006).

Indivíduos portadores de Síndrome de Down (SD) dentre todas as Deficiências Mentais (DM), é a única população que compõem um grupo onde se observam prevalências de excesso de peso e obesidade superiores às encontradas em populações adultas saudáveis, o que aumenta o risco de infarto agudo do miocárdio, hipertensão arterial e diabetes, conduzindo a uma menor expectativa de vida (SILVA et al., 2006).

Isso ocorre, principalmente, devido às alterações gênicas encontradas em indivíduos com SD que por si só são suficientes para induzir maior incapacidade física, que pode estar relacionada com a menor disposição desse grupo populacional em relação à atividade física, contribuindo com o aumento significativo de tecido adiposo. Assim como, o ganho de peso está relacionado ao meio e hábitos de vida em que o portador da SD está inserido (SILVA et al., 2006).

Em estudo realizado com o objetivo de caracterizar uma amostra de 104 indivíduos da população portuguesa portadora de $\mathrm{SD}$, quanto à composição corporal, segundo o gênero e a idade. Notou-se ao se comparar os portadores de SD com um grupo controle, uma maior prevalência de excesso de peso e de obesidade em homens e mulheres com SD, com uma maior incidência de obesidade nas mulheres, não encontrando, no entanto, diferenças significativas em relação às faixas etárias entre os gêneros (SILVA et al., 2006). 
Para medir as pressões respiratórias, utilizou-se o manovacuômetro digital, que tem por finalidade medir pressões positivas (manômetro) e negativas (vacuômetro), que permite a leitura e armazenamento de pressões máximas de inspiração (Pi max) e expiração (Pe max), previamente calibrado. A Pi max foi medida durante um esforço iniciado a partir do volume residual e a Pe max foi medida durante um esforço iniciado a partir da capacidade pulmonar total (BIANCHI, 2009).

Quando se comparou a Pi max prevista com a Pi max obtida observou-se diferença significativa $(\mathrm{p}=0,000)$ entre as variáveis.

São várias as alterações morfológicas do pulmão que comprometem diretamente a funcionalidade de todo o sistema respiratório, dentre elas citam-se: o alargamento dos ductos alveolares, a diminuição do número dos alvéolos, a presença de uma camada capilar dupla na maioria das paredes alveolares, a falta de crescimento radial do tecido pulmonar após o nascimento, fato ainda em estudo hoje em dia, onde se supõem que seja devido a alguma falha genética. Além disso, essas crianças possuem uma maior predisposição a infecções, particularmente da árvore respiratória, devido, principalmente, às alterações imunes que irão consequentemente levar a alterações pulmonares e respiratórias comprometendo a biomecânica respiratória e as trocas gasosas além do aporte de oxigênio disponibilizado para todos os órgãos e tecidos, como o diafragma, que ficará prejudicado funcionalmente devido ao baixo aporte de nutrientes e oxigênio, transformando o comprometimento do sistema respiratório um ciclo vicioso e prejudicial a todo o organismo que se encontra em uma situação de baixas pressões inspiratórias (SCHWARTZMAN, 2003).

Os dados deste estudo apontam que indivíduos com Síndrome de Down apresentam redução da Pe max, quando comparados os valores obtidos com os previstos, apresentando significância estatística, sendo $(\mathrm{p}=0,000)$. As pressões respiratórias dos indivíduos portadores de SD podem ser afetadas por todos os fatores citados anteriormente. Podendo gerar fraqueza da musculatura respiratória, principalmente os abdominais observada na diminuição da Pe max, que levariam como resultado a uma diminuição da eficácia da tosse, causando acúmulo de secreções e o aparecimento de pneumonias (CASTOLDI et al., 2012).

Indivíduos com SD podem apresentar baixos valores de pressões respiratórias, sendo que os valores da Pe max costumam ficar abaixo de 50\% e de Pi max abaixo de $60 \%$ do previsto para a população jovem sedentária saudável. Isso ocorre devido a uma diminuição da complacência pulmonar, que leva ao colapso das unidades pulmonares distais, devido principalmente à incapacidade de suspirar ou respirar profundamente dos portadores de SD, 
assim como por apresentarem uma tosse ineficaz que possibilita a retenção de secreções e o aparecimento de pneumonias (CASTOLDI et al., 2012).

Supõe-se que a alteração da Pe max aqui observada seja principalmente decorrente de características particulares da SD, presentes em aproximadamente em 100\% dos portadores, que é a hipotonia muscular, assim como o acúmulo de gordura abdominal.

Com a deposição crescente de gordura, revestindo a cavidade torácica em indivíduos com sobrepeso, tanto dentro como sobre a cavidade abdominal leva a progressivas alterações na função respiratória dentre essas, as mais importantes, estão a diminuição da Capacidade Residual Funcional (CRF), causada pelo processo mecânico simples de "compressão" da cavidade torácica, e uma consequente redução nas dimensões anatômicas pela massa de tecido adiposo de revestimento. Em indivíduos com acúmulo de tecido adiposo o diafragma encontra-se mais elevado pelo abdômen distendido. A redução da CRF ocorre tanto pela redução do Volume de Reserva Expiratória (VRE) como pela redução do Volume Residual (VR). Considerando que a redução do VRE pode causar anormalidades na relação ventilação/perfusão, nos gases do sangue arterial, nos mecanismos pulmonares e na difusão dos gases. Essa condição de sobrepeso eleva o custo de oxigênio da respiração, isso ocorre porque os músculos intercostais movimentam uma massa aumentada do tórax, o diafragma contrai e desce, para atuar contra a pressão de um abdômen aumentado como ocorre em indivíduos obesos (FUNG et al., 1989).

Outro fator que poderia estar relacionado à diminuição da Pe max, é a hipotonia muscular que pode ser classificada em dois subtipos: a hipotonia intrínseca ou primária, que está relacionada com o acometimento das unidades motoras periféricas e a hipotonia secundária, que, por sua vez, ocorre principalmente como sinal acessório em desordens neurológicas com comprometimento do sistema nervoso central, ou no contexto de síndromes genéticas, a qual se engloba a SD, patologia em questão discutida neste trabalho, bem como de doenças sistêmicas graves de caráter extra neurológico (REED, 2002).

Ao se correlacionar os coeficientes de correlação (R) e os valores de significância (p) entre as variáveis Pi max obtida, Pe max obtida, observa-se que não houve correlação entre nenhuma das correlações aqui abordadas.

Assim sendo os valores da Pi max, Pe max não foram suficientemente satisfatórios para influenciar na mecânica respiratória.

Quando se correlacionou os dados: IMC, IDADE com Pi max obtida, Pe max obtida, observou correlação apenas entre as variáveis IMC e Pe max obtida. 
O aumento da deposição de tecido adiposo, assim como já analisada anteriormente, está relacionado com o comprometimento da força muscular de todo o organismo bem como da musculatura encarregada pela dinâmica respiratória, que irá ser prejudicada quanto à função de disponibilizar oxigênio, necessário para a realização de todas as atividades.

Tal correlação existente entre o IMC e a Pe max pode ser justificada, pois com o aumento da deposição de gordura na região torácica e abdominal, ocorre consequentemente a diminuição da mobilidade da parede do tórax e abdômen, fazendo com que a complacência torácica diminua e o trabalho respiratório eleve-se, devido à redução da força dos músculos intercostais externos e abdominais que se encontram distendidos, dificultando a relação ventilação - perfusão comprometendo a funcionalidade de vários sistemas corporais, assim como comprometendo a qualidade de vida do indivíduo (SOARES et al., 2011).

Este estudo apresentou algumas possíveis limitações. Primeiramente, a dificuldade na captação de indivíduos dispostos a permitirem a participação de seus filhos na pesquisa, assim como a compreensão dos indivíduos portadores da síndrome e execução de todos os testes necessários para o desenvolvimento do projeto, onde o número reduzido da amostra pode ter interferido na estatística e ser responsável pela ausência de algumas associações. No entanto, várias associações foram aqui levantadas, respondendo às intenções iniciais do presente estudo.

Outra limitação encontrada foi relacionada à escassez na literatura sobre a força muscular respiratória em indivíduos com SD e sabe-se de sua importância para o conhecimento prévio para identificação de possíveis alterações, prevenção de complicações e consequente melhora da qualidade de vida de tais indivíduos.

Diante disso, sugere-se a realização de novos estudos para complementação do conhecimento na área, realizando-se com um grupo amostral composto por maior número de indivíduos portadores de SD, onde este apresente uma composição mais homogênea para que seja confirmada a hipótese de que a amostra aqui apresentada tenha sofrido alguma influência, e também estudos onde seja possível comparar os gêneros (masculino e feminino) para verificar se existe alguma diferença entre a força muscular respiratória entre ambos. 


\section{REFERÊNCIAS}

AQUINO, E. da S. et al. Correlação entre a composição corporal e força, resistência da musculatura respiratória e capacidade de exercício em pacientes com doença pulmonar obstrutiva crônica. Revista Fisioterapia e Pesquisa, São Paulo, v. 17, n. 1, jan./mar. 2010.

BERTHOLD, B. T. et al. Síndrome de Down: aspectos gerais e odontológicos. Revista Ciências Médicas e Biológicas, Salvador, v. 3, n. 2, p. 252-260, jul./dez. 2004.

BIANCHI, P. D. A. et al. Avaliação do treinamento de força e resistência muscular respiratória com manovacuômetro e com carga linear pressórica. Revista Digital, Buenos Aires, v. 14, n. 136, 2009.

CASTOLDI, A. et al. Avaliação da força muscular e capacidade respiratória em pacientes com Síndrome de Down após Bad Ragaz. Revista Neurociências, São Paulo, v. 20, n. 3, p. 386-391, 2012.

FUNG, K. P. et al. Effects of overweight on lung function. American Review of Respiratory Disease, v. 128, p. 512-515, 1989.

GRANZOTTI, J. A. et al. Incidência de cardiopatias congênitas na Síndrome de Down. Jornal de Pediatria, Rio de Janeiro, v. 71, n. 1, p. 28-30, 1995.

GUSMÃO, F. A. F.; TAVARES, E. J. M.; MOREIRA, L. M. A. Idade materna e Síndrome de Down no Nordeste do Brasil. Cadernos de Saúde Pública, Rio de Janeiro, v. 19, n. 4, p. 973978, jul./ago. 2003.

JUNIOR, L. A. F. et al. Avaliação da força muscular respiratória e da função pulmonar em pacientes com insuficiência cardíaca. Arquivos Brasileiros de Cardiologia, São Paulo, v. 89, n. 1, jul. 2007.

MOREIRA, L. M. A.; EL-HANI, C. N.; GUSMÃO, F. A. F. A Síndrome de Down e sua patogênese: considerações sobre o determinismo genético. Revista Brasileira de Psiquiatria, São Paulo, v. 22, n. 2, p. 96-9, 2000.

PEDROSA, R.; HOLANDA, G. Força muscular respiratória e capacidade funcional em idosas hipertensa com sonolência diurna excessiva. Revista Fisioterapia e Pesquisa, São Paulo, v. 17, n. 2, abr./jun. 2010.

REED, U. C. Doenças neuromusculares. Jornal de Pediatria, Rio de Janeiro, v. 78, supl. 1, jul/ago. 2002.

RUIVO, S.; VIANA, P.; MARTINS, C.; BAETA, C. Efeito do envelhecimento cronológico na função pulmonar. Comparação da função respiratória entre adultos e idosos saudáveis. Revista Portuguesa de Pneumologia, ago. 2009.

SCHWARTZMAN, J. S. Síndrome de Down. 2. ed. São Paulo: Mamnon, 2003.

SILVA, D. L. et al. Avaliação da composição corporal em adultos com Síndrome de Down. Arquivos de Medicina, Porto, Portugal, v. 20, n. 4, p. 103-10, 2006. 
SILVA, N. L. P.; DESSEN, M. A. Síndrome de Down: etiologia, caracterização e impacto na família. Interação em Psicologia, Curitiba, v. 6, n. 2, p. 167-176, 2002.

SILVA, V. R. Aspectos clínicos da Síndrome de Down. Londrina: Centro de Especialização em Fonoaudiologia Clinica (CEFAC), 2000.

SOARES, J. A. et al. Distúrbios respiratórios em crianças com síndrome de Down. Arquivos de Ciência da Saúde, São José do Rio Preto, v. 11, n. 4, p. 230-3, out./dez. 2004.

SOARES, K. K. D. et al. Avaliação do desempenho físico e funcional respiratório em obesos. Fisioterapia em Movimento, Curitiba, v. 24, n. 4, out./dez. 2011.

TAMASHIRO, E. et al. Efeitos do cigarro sobre o epitélio respiratório e sua participação na rinossinusite crônica. Jornal Brasileiro de Otorrinolaringologia, São Paulo, v. 75, n. 6, nov./dez. 2009. 